\title{
Optimization of an HPLC Method for Determination of Gabapentin in Dosage Forms through Derivatization with 1-Fluoro-2,4-dinitrobenzene
}

\author{
Effat Souri, * Hassan Jalalizadeh, and Abbas Shafiee \\ Department of Medicinal Chemistry, Faculty of Pharmacy and Pharmaceutical Sciences Research Center, Medical \\ Sciences/University of Tehran; Tehran 14155-6451, Iran. Received February 17, 2007; accepted July 1, 2007
}

\begin{abstract}
A rapid, sensitive and accurate high performance liquid chromatography with $U V$ detection method was developed and validated for the quantification of gabapentin in dosage forms. Gabapentin was quantified after precolumn derivatization with 1-fluoro-2,4-dinitrobenzene. Amlodipine was used as an internal standard. The chromatographic separation was carried out on a Nova-Pak $\mathrm{C}_{18}$ column using a mixture of acetonitrile-sodium dihydrogenphosphate $(\mathrm{pH} 2.5 ; 0.05 \mathrm{M})(70: 30, \mathrm{v} / \mathrm{v})$ as mobile phase with $\mathrm{UV}$ detection at $360 \mathrm{~nm}$. The method was linear over the range of $10-500 \mu \mathrm{g} / \mathrm{ml}$ of gabapentin $\left(r^{2}>0.999\right)$. The within-day and between-day precision values were in the range of $0.86-1.11 \%$. The method was successfully used for quantitative determination and dissolution rate study of Neurontin capsules.
\end{abstract}

Key words gabapentin; 1-fluoro-2; 4-dinitrobenzene; HPLC; UV-detection; dosage form

Gabapentin, 1-(aminomethyl)-cyclohexaneacetic acid, (Fig. 1) which is a structural analogue of the inhibitory neurotransmitter $\gamma$-aminobutyric acid (GABA), is a potent antiepileptic drug. Gabapentin is used for the treatment of complex partial seizures, with or without secondary generalization in patients over 12 years of age. ${ }^{1)}$ The mechanism of action of gabapentin is not completely understood. Recently, it has been shown that the action of gabapentin is possibly due to its high binding to $\alpha_{2}-\delta$ protein, an auxiliary subunit of voltage-gated calcium channels. Potent binding at this site reduces the release of several neurotransmitters which would reduce neuronal excitability and seizures. ${ }^{2)}$

Gabapentin has no significant ultraviolet, or visible absorption, or fluorescence. Derivatization by chromophoric reagents increases the sensitivity of gabapentin detection. Several HPLC methods for determination of gabapentin in human plasma have been published using different derivatizing reagents such as 2,4,6-trinitrobenzenesulphonic acid, ${ }^{3,4}$ ) phenylisothiocyanate, ${ }^{5)} o$-phthaldialdehyde (OPA), ${ }^{6-12)} 4$ chloro-7-nitrobenzofurazan, ${ }^{13)}$ 9-fluorenylmethyl chloroformate $^{14)}$ and 1,2-naphthoquinone-4-sulphonic acid sodium salt. ${ }^{15)}$ Most of the proposed methods could be optimized for determination of gabapentin in dosage forms but they suffer from some limitations such as a lengthy run time or using

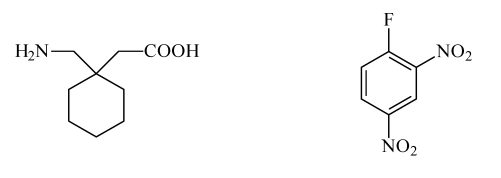

Gabapentin 1-Fluoro-2,4-dinitrobenzene

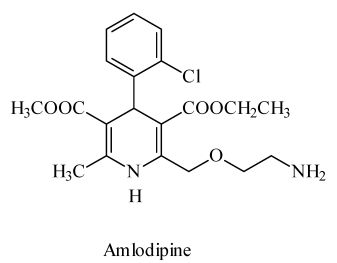

Fig. 1. Chemical Structure of Gabapentin, 1-Fluoro-2,4-dinitrobenzene and Amlodipine (I.S.) special reaction conditions which may not be suitable for routine analysis. OPA was used in most published methods. $^{6-12)}$ Using OPA as a derivatizing reagent, the fluorescent OPA-drug derivative should be injected immediately after preparation because of the instability of the adduct. Therefore, the method is difficult to apply for routine studies especially when automated instrumentation is not available. Derivatization with phenylisothiocyanate (PITC) is simple, but this reagent degrades in contact with water and the reaction medium should be completely free from water before the addition of reagent. ${ }^{5)}$ Using 4-chloro-7-nitrobenzofurazan as a labeling reagent, the adduct should be extracted and detected by spectrofluorimetry. ${ }^{13}$ ) The reaction time for gabapentin and 9-fluorenylmethyl chloroformate is not too long (about $10 \mathrm{~min}$ ), but a column temperature of $60^{\circ} \mathrm{C}$ is needed for separation of the adduct. ${ }^{14)}$ In the method reported by Sagirli et al. $^{15)}$ derivatization with 1,2-naphthoquinone-4-sulphonic acid and extraction of the reaction product were needed and the total run time of the HPLC method was $15 \mathrm{~min}$.

Currently none of the gabapentin dosage forms are indexed in any pharmacopoeia. A direct HPLC method at $215 \mathrm{~nm}$ is reported for analysis of gabapentin in bulk drug in the 2007 USP. $^{16)}$ To the best of our knowledge, few methods have been reported in the literature for determination of gabapentin in pharmaceutical formulations. In one of the methods, spectrofluorometric determination of gabapentin was reported after derivatization with fluorescamine. ${ }^{17)}$ In another study, colorimetric determination of gabapentin was studied based on the reaction with vanillin and ninhydrin. ${ }^{18)}$ In these cases, the derivatization condition was time consuming and the stability of the reaction products depends on experimental conditions such as $\mathrm{pH}$, temperature and reaction time. A capillary electrophoresis method was also reported for determination of gabapentin in bulk drug and capsules. ${ }^{19)}$

The aim of the present study was to develop a rapid, accurate and precise HPLC method for determination of gabapentin following derivatization with 1-fluoro-2,4-dinitrobenzene (FDNB) (Fig. 1) in pharmaceutical formulations and in dissolution media for drug quality control purposes. Recently, FDNB has been used for HPLC determination of vertilmicin in rat serum, ${ }^{20)}$ amikacin in the skin ${ }^{21)}$ and spec- 
trophotometric determination of lisinopril in tablets. ${ }^{22)}$

\section{Experimental}

Materials Gabapentin (Ranbaxy, India, Batch No: GIN0605006) was a kind gift from Daru-Darman Pars Company (Tehran, Iran). Amlodipine besylate (internal standard, I.S.) (Fig. 1) was obtained from Eczacibasi (Istanbul, Turkey). Neurontin 100 capsules (Pfizer, France, Batch No: 0022035) were purchased from a local pharmacy. 1-Fluoro-2,4-dinitrobenzene (purity $>99 \%$ ) (FDNB) was purchased from Fluka (Switzerland). Acetonitrile was HPLC grade and purchased from Merck (Darmstadt, Germany). All other chemicals were of analytical grade and used without any further purification.

Instrumentation A SHIMADZU UV-160 double beam UV-visible spectrophotometer (Shimadzu, Kyoto, Japan) with a fixed bandwidth $(2 \mathrm{~nm})$ and $1-\mathrm{cm}$ quartz cell was used.

The HPLC system consisted of a 600 Pump, 710 plus Autosampler, and a variable 480 UV Detector all from Waters (Milford, MA, U.S.A.). The data processing system was a multi-channel Chrom \& Spec software for chromatography, version $1.5 \mathrm{X}$.

Standard Solutions Stock standard solution of gabapentin was prepared by dissolving an appropriate amount of the compound in distilled water to give a final concentration of $1000 \mu \mathrm{g} / \mathrm{ml}$. Standard solutions of gabapentin $(10,20,50,100,200,300,400,500 \mu \mathrm{g} / \mathrm{ml})$ were prepared by subsequent dilution. A solution of I.S. was prepared by dissolving amlodipine besylate in a mixture of acetonitrile and water $(20: 80)$ to a final concentration of $150 \mu \mathrm{g} / \mathrm{ml}$. Stock solutions of FDNB $(0.012 \mathrm{M})$ were prepared by dissolving $150 \mu \mathrm{l}$ of the reagent in $100 \mathrm{ml}$ of acetonitrile. This reagent should be handled carefully since it is a skin irritant. All these solutions were stored at $4{ }^{\circ} \mathrm{C}$.

A $0.25 \mathrm{M}$ borate buffer containing $0.25 \mathrm{M} \mathrm{KCl}$ was prepared by dissolving appropriate amounts of $\mathrm{H}_{3} \mathrm{BO}_{3}$ and $\mathrm{KCl}$ in water and adjusting the $\mathrm{pH}$ to 8.2 by adding $2 \mathrm{~m} \mathrm{NaOH}$, while checking the $\mathrm{pH}$ with a $\mathrm{pH}$ meter.

A $1 \mathrm{~m}$ hydrochloric acid solution was prepared by diluting a $12 \mathrm{M}$ hydrochloric acid commercial solution.

Sample Preparation Into $10 \mathrm{ml}$ measuring flasks, $1 \mathrm{ml}$ of each standard solution of gabapentin and $0.5 \mathrm{ml}$ of amlodipine solution (I.S.) were transferred. To each flask, $1 \mathrm{ml}$ borate buffer, $400 \mu \mathrm{l}$ of FDNB reagent and $3.6 \mathrm{ml}$ of acetonitrile were added. After $2 \mathrm{~s}$ vortexing, the flasks were kept at $65^{\circ} \mathrm{C}$ for $10 \mathrm{~min}$. After cooling to room temperature, $150 \mu \mathrm{l}$ of $1 \mathrm{~m} \mathrm{HCl}$ solution was added and the volumetric flasks brought up to volume with acetonitrile. Twenty microliters of the resulting solution was injected into the HPLC system.

Chromatographic Conditions Separation was achieved using a Nova$\mathrm{Pak}^{\circledR} \mathrm{C}_{18} 4 \mu \mathrm{m}$ column $(250 \mathrm{~mm} \times 4.6 \mathrm{~mm}$, Waters, Milford, MA, U.S.A. $)$ The isocratic mobile phase pumped at a flow-rate of $1.5 \mathrm{ml} / \mathrm{min}$ consisted of acetonitrile-sodium dihydrogenphosphate $(\mathrm{pH} 2.5 ; 0.05 \mathrm{M})(70: 30, \mathrm{v} / \mathrm{v})$ prepared daily and degassed by passing through a $0.45 \mu \mathrm{m}$ Millipore filter and ultrasonication for $10 \mathrm{~min}$. All separations were performed at room temperature with detection at $360 \mathrm{~nm}$.

Optimization of Reaction Conditions The derivatization of gabapentin with FDNB was optimized using solutions of $500 \mu \mathrm{g} / \mathrm{ml}$ of the drug. In order to optimize the reaction conditions, various amounts of FDNB reagent ranging from $100-1000 \mu \mathrm{l}$ were studied at two different temperatures $\left(65^{\circ}\right.$, $90^{\circ} \mathrm{C}$ ) and different reaction times. Optimized conditions were obtained when $400 \mu \mathrm{l}$ of the reagent solution was used and the reaction mixture was kept at $65^{\circ} \mathrm{C}$ for $10 \mathrm{~min}$.

Calibration Six series of standard calibration solutions were prepared by standard solutions of gabapentin and $0.5 \mathrm{ml}$ of I.S. solution. The sample

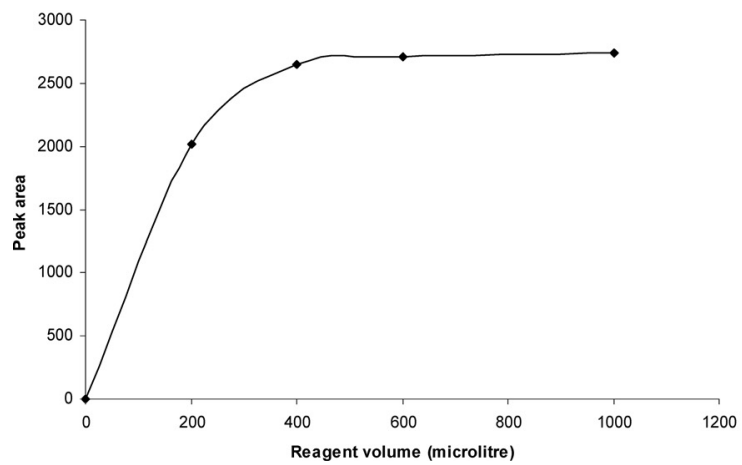

preparation and HPLC analysis were performed as described above. Calibration curves were constructed by plotting the measured peak area ratios of gabapentin to the I.S. versus concentrations of standard samples and then statistical analysis was performed.

Precision and Accuracy In order to evaluate the within-day and between-day precision and accuracy, three replicates of standard solutions at three different concentrations $(10,100,500 \mu \mathrm{g} / \mathrm{ml})$ were assayed on one day and three separate days.

Application of the Method The contents of 20 Neurontin capsules were combined and weighed. An amount of powder equivalent to about $100 \mathrm{mg}$ of gabapentin was accurately weighed, transferred to a $100 \mathrm{ml}$ volumetric flask, made up to volume with distilled water and placed in an ultrasonic bath for $15 \mathrm{~min}$. After passing through a $0.45 \mu \mathrm{m}$ Millipore filter, the solution was diluted with water to obtain a concentration of about $150 \mu \mathrm{g} / \mathrm{ml}$. The drug concentrations of six replicates were determined by HPLC using the calibration curve.

Dissolution Studies Drug release was measured using a dissolution apparatus (Erweka, Heusenstamm, Germany). The apparatus consists of six vessels in a warm bath at $37^{\circ} \mathrm{C}$. The dissolution medium was $900 \mathrm{ml}$ of deionized, freshly prepared water. The paddle apparatus was used for capsules and the rotation speed was kept at $50 \mathrm{rpm}$. To avoid floating, a ring/mesh stainless steel device, which fits under the paddle into the lower portion of the dissolution vessel, was employed. For each dissolution profile, one capsule was added to the medium and samples of $4 \mathrm{ml}$ were drawn at 5 , 10,20 , and $30 \mathrm{~min}$. Sample volumes were replaced by fresh dissolution media prewarmed at $37^{\circ} \mathrm{C}$ to maintain a constant total volume. The dissolution vessels were covered to minimize evaporation. The solutions were passed through a $0.45 \mu \mathrm{m}$ Millipore filter and treated according to the above mentioned method.

\section{Results and Discussion}

Derivatization Reaction In the present method, pre-column derivatization with FDNB was used for the analysis of gabapentin. Reaction of FDNB with primary amines is carried out in the presence of borate buffer $(\mathrm{pH}=8.2)$. A hydrolysis product of FDNB, dinitrophenolale, is also formed in the reaction mixture which turns to dinitrophenol after acidification at the end of derivatization. ${ }^{22)}$ The UV absorption spectra of the gabapentin-FDNB derivative showed a maximum at $360 \mathrm{~nm}$ which was used as the HPLC analytical wavelength.

Using different amounts of the reagent, it was shown that by increasing the amount of the reagent, the peak area of the gabapentin derivative was increased. The completion of the reaction was achieved using $400 \mu \mathrm{l}$ of the reagent (Fig. 2). Larger amounts of reagent did not show any effect on reaction yield but increased the proportion of interfering byproducts. By studying different reaction temperatures, it was found that the reaction was completed after $10 \mathrm{~min}$ at $65^{\circ} \mathrm{C}$ or $5 \mathrm{~min}$ at $90^{\circ} \mathrm{C}$ (Fig. 2). The reaction product was not stable at $90^{\circ} \mathrm{C}$, whereas its degradation proceeded slowly at

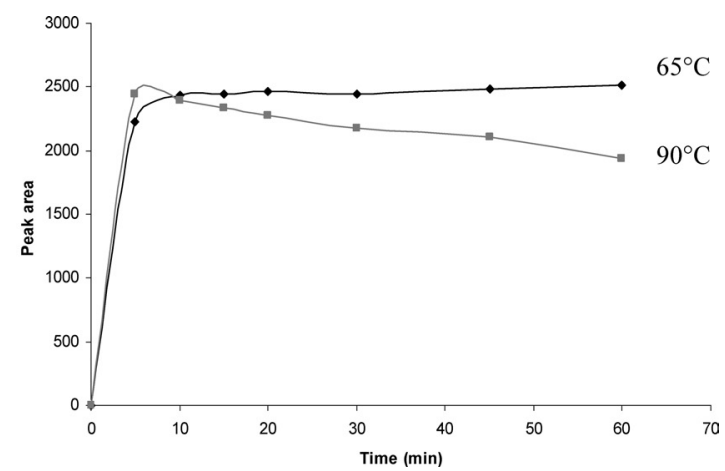

Fig. 2. Effect of Reagent Amount $\left(65^{\circ} \mathrm{C}\right.$ for $\left.10 \mathrm{~min}\right)$, Time and Temperature on the Peak Area of the FDNB-Gabapentin Derivative ( $\left.n=3\right)$ 

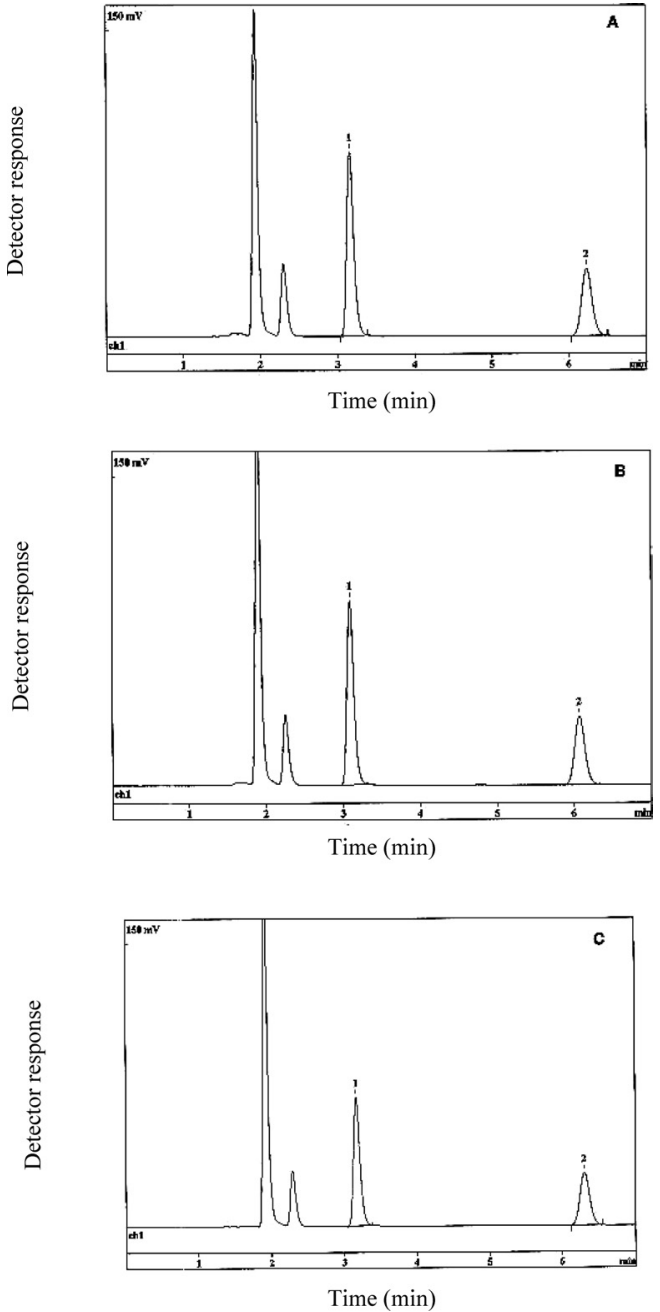

Fig. 3. HPLC Chromatograms of Gabapentin $(100 \mu \mathrm{g} / \mathrm{ml})$ and I.S. Standard Solutions (A); Assay Preparation of Neurontin (B); Test Solution of Dissolution Medium after 5 min (C)

Peaks: 1, gabapentin; 2, I.S.

lower temperature and looked stable. No reaction was achieved at room temperature. The best results were obtained at $65^{\circ} \mathrm{C}$ within $10 \mathrm{~min}$.

Chromatographic Conditions Optimization of chromatographic conditions was achieved by monitoring varying columns and mobile systems. Silica columns such as a $\mu$ Bondapak column with different mobile phases did not give a suitable peak shape or separation of peaks for analysis. On the other hand, Nova-Pak $\mathrm{C}_{18}$ gave better results. After trying different ratios of mixtures of methanol-phosphate buffer and acetonitrile-phosphate buffer, the best results were achieved by using a mixture of acetonitrile-sodium dihydrogenphosphate $(\mathrm{pH} 2.5 ; 0.05 \mathrm{M})(70: 30, \mathrm{v} / \mathrm{v})$ as mobile phase. Excellent chromatographic specificity with no interference from the reagent by-products or dosage form excipients was observed. Moreover, suitable retention times for gabapentin and amlodipine derivatives were achieved. Typical chromatograms obtained from the standard solution of gabapentin, assay preparation of Neurontin capsules and a test solution from dissolution medium of Neurontin capsules are presented in Fig. 3. Under the chromatographic conditions described, gabapentin and I.S. were well resolved and eluted
Table 1. Statistical Data of Calibration Curves of Gabapentin in Standard Solutions

\begin{tabular}{lc}
\hline \hline \multicolumn{1}{c}{ Parameters } & Gabapentin \\
\hline Linearity & $10-500 \mu \mathrm{g} / \mathrm{ml}$ \\
Regression equation & $Y=0.0185 x-0.027$ \\
Standard deviation of slope & 0.00014 \\
Relative standard deviation of slope $(\%)$ & 0.74 \\
Standard deviation of intercept & 0.027 \\
Correlation coefficient $\left(r^{2}\right)$ & 0.9998 \\
Standard deviation of residuals & 0.018 \\
\hline
\end{tabular}

Table 2. Precision and Accuracy of Method for Determination of Gabapentin in Standard Solutions ( $n=9 ; 3$ Sets for $3 \mathrm{~d})$

\begin{tabular}{ccrr}
\hline \hline $\begin{array}{c}\text { Concentration added } \\
(\mu \mathrm{g} / \mathrm{ml})\end{array}$ & $\begin{array}{c}\text { Concentration calculated } \\
(\text { mean } \pm \text { S.D. })(\mu \mathrm{g} / \mathrm{ml})\end{array}$ & CV $(\%)$ & Error $(\%)$ \\
\hline Within-day $(n=3)$ & & & \\
10.00 & $10.16 \pm 0.09$ & 0.86 & 1.60 \\
100.00 & $99.32 \pm 1.07$ & 1.07 & -0.68 \\
500.00 & $496.15 \pm 4.85$ & 0.98 & -0.77 \\
Between-day $(n=9)$ & & & \\
10.00 & $10.07 \pm 0.09$ & 0.92 & 0.70 \\
100.00 & $99.57 \pm 1.11$ & 1.11 & -0.43 \\
500.00 & $499.98 \pm 5.01$ & 1.00 & -0.00 \\
\hline
\end{tabular}

at about 3 and $6 \mathrm{~min}$, respectively and the total run time was within $7 \mathrm{~min}$. Good baseline resolution and peak shape can be observed.

Linearity Calibration curves were constructed using six series of standard gabapentin solutions in the range of 10 $500 \mu \mathrm{g} / \mathrm{ml}$. A linear relationship was obtained between the peak area ratio of gabapentin to that of I.S. versus the corresponding concentration, as shown by the equation presented in Table 1. Statistical data are presented in Table 1.

Accuracy and Precision The accuracy and precision were determined by analyzing three synthetic samples of gabapentin at 10,100 and $500 \mu \mathrm{g} / \mathrm{ml}$ on three separate days. Within-day and between-day data are given in Table 2. Good accuracy and repeatability were observed over the entire concentration range. The within-day and between-day variability showed $\mathrm{CV}$ values less than $1.2 \%$ in all three selected concentrations. The intermediate precision was assessed by comparison of the within-day and between-day data for analysis of gabapentin samples by two analysts using two different HPLC systems. The CV values did not exceed $2 \%$.

Also, the influences of small changes in the mobile phase composition $( \pm 10 \%)$ and buffer $\mathrm{pH}( \pm 0.3)$ were studied to determine the robustness of the method, such as the changes in peak area and retention time. Peak area values were not influenced (less than $\pm 0.17 \%$ ) by changing the composition of mobile phase or $\mathrm{pH}$ of the phosphate buffer. The retention time of gabapentin was negligibly influenced $(1.6 \%)$ by changing the $\mathrm{pH}$ of the phosphate buffer, whereas it was slightly influenced but less than $9.5 \%$ by changing the mobile phase composition. The results are summarized in Table 3.

Specificity The specificity test of the proposed method demonstrated that the excipients from capsules did not interfere in the drug or I.S. peak. Furthermore, well-resolved peaks indicated the specificity of the method. Thus the pro- 
Table 3. The Influence of Small Changes in $\mathrm{pH}$ and Composition of Mobile Phase (Method Robustness)

\begin{tabular}{ccc}
\hline \hline Mobile phase composition & $t_{\mathrm{R}}$ & Peak area \\
\hline Acetonitrile-buffer $\mathrm{pH}=2.3(73: 27)$ & 2.76 & 592.29 \\
Acetonitrile-buffer $\mathrm{pH}=2.3(70: 30)$ & 2.99 & 593.56 \\
Acetonitrile-buffer $\mathrm{pH}=2.3(67: 33)$ & 3.28 & 588.11 \\
Acetonitrile-buffer $\mathrm{pH}=2.5(73: 27)$ & 2.79 & 607.02 \\
Acetonitrile-buffer $\mathrm{pH}=2.5(70: 30)$ & 3.03 & 607.02 \\
Acetonitrile-buffer $\mathrm{pH}=2.5(67: 33)$ & 3.33 & 591.20 \\
Acetonitrile-buffer $\mathrm{pH}=2.7(73: 27)$ & 2.71 & 605.31 \\
Acetonitrile-buffer $\mathrm{pH}=2.7(70: 30)$ & 2.96 & 598.84 \\
Acetonitrile-buffer $\mathrm{pH}=2.7(67: 33)$ & 3.24 & 593.62 \\
\hline
\end{tabular}

$t_{\mathrm{R}}:$ retention time $(\min )$

Table 4. System Suitability Parameters

\begin{tabular}{lcl}
\hline \multicolumn{1}{c}{ Parameter } & Found & Acceptable limits \\
\hline Theoretical plates $(n=3)$ & 2200 & $\mathrm{~N}>1500$ \\
Asymmetry $(n=6)$ & 0.99 & $\mathrm{~T}<1.5$ \\
Repeatability $\left(t_{\mathrm{R}}\right)(n=6)$ & 0.30 & R.S.D. $<1 \%$ \\
Repeatability (peak area) $(n=6)$ & 0.56 & R.S.D. $<1 \%$ \\
\hline
\end{tabular}

$t_{\mathrm{R}}$ : retention time $(\min ) ; \mathrm{N}$ : theoretical plates; T: tailing factor; R.S.D.: relative standard deviation.

posed HPLC method was useful to quantify gabapentin in dosage forms.

Sensitivity The limit of quantification with CV $<1.2 \%$ was found to be $10 \mu \mathrm{g} / \mathrm{ml}$ for gabapentin. The limit of detection with an $\mathrm{S} / \mathrm{N}$ ratio of 3 was found to be $3 \mu \mathrm{g} / \mathrm{ml}$.

System Suitability Test System suitability testing was performed to verify the repeatability of the HPLC method. Theoretical plates, symmetry and repeatability of the retention time and peak area were determined and compared. The results and the limits are summarized in Table 4.

Solution Stability The stability of the stock solution was determined by analyzing gabapentin in comparison to freshly prepared standard solution. No significant changes $(<1 \%)$ were observed in stock solution after $7 \mathrm{~d}$ in comparison to freshly prepared standard. The gabapentin derivative was also stable for at least $24 \mathrm{~h}$.

Assay and Dissolution Test of Neurontin Capsules The developed method was applied to quantitative determination of Neurontin capsules. The results were shown to be in good agreement with the labeled amount $(96.34 \pm 0.59 \mathrm{mg})$ and the error of the determination did not exceed $\pm 3.8 \%$.

The dissolution profile of Neurontin capsules obtained with the described method is presented in Fig. 4. It was observed that more than $90 \%$ of the drug was dissolved within $10 \mathrm{~min}$.

\section{Conclusion}

In conclusion, the proposed HPLC method provided a simple, accurate and reproducible method for routine in vitro tests of gabapentin dosage forms. Although several HPLC methods are now available for determination of gabapentin with UV detection, this is the first report for using FDNB as a derivatizing reagent without the limitations of the previously reported methods. The major advantages of this method include the use of an inexpensive derivatizing

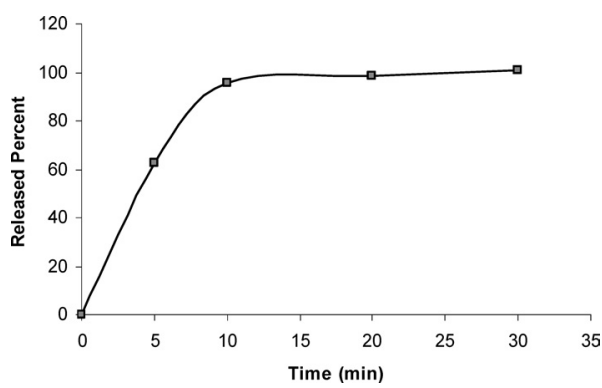

Fig. 4. Dissolution Profile of $100 \mathrm{mg}$ Neurontin Capsules $(n=6)$ Using Water as Dissolution Medium and Paddle at $50 \mathrm{rpm}$

reagent, short derivatization time, stability of the reaction product, no need for prior separation or purification before analysis, and the applicability of a common HPLC system (isocratic system, UV detector and ambient temperature). The short chromatographic time makes this method suitable for the processing of multiple samples in a limited amount of time. Finally, since no pharmacopoeial method for determination of gabapentin in pharmaceutical dosage forms and dissolution medium has been reported yet, the proposed method could be useful and suitable for quality control studies.

Acknowledgement The authors would like to thank the Iran National Science Foundation for the financial support of this research project.

\section{References}

1) Tallian K. B., Nahata M. C., Lo W., Tsao C. Y., J. Clin. Pharm. Ther, 29, 511-515 (2004).

2) Taylor C. P., Angelotti T., Fauman E., Epilepsy Res., 73, 137-150 (2007).

3) Hengy H., Kolle E. U., J. Chromatogr. B, 341, 473-478 (1985).

4) Juenke J. M., Brown P. I., McMillin G. A., Urry F. M., Clin. Chem., 49, 1198-1201 (2003).

5) Zhu Z., L. Neirinck L., J. Chromatogr. B, 779, 307-312 (2002).

6) Forrest G., Sills G. L., Leach J. P., Brodie M. J., J. Chromatogr. B, 681, 421-425 (1996).

7) Ratnaraj H., Patsalos P. N., Ther. Drug Monit., 20, $430-434$ (1998)

8) Jiang Q., Li S., J. Chromatogr. B, 727, 119-123 (1999).

9) Tang P. H., Miles M. V., Glauser T. A., De Grauw T., J. Chromatogr. B, 727, 125-129 (1999).

10) Chollet D. F., Goumaz L., Juliano C., Anderegg G., J. Chromatogr. B, 746, 311-314 (2000).

11) Gauthier J. D., Gupta R., Clin. Chem., 48, 2259-2261 (2002).

12) Vermeij T. A. C., Edelbroek P. M., J. Chromatogr. B, 810, 297-303 (2004).

13) Bahrami Gh., Mohammadi B., J. Chromatogr. B, 837, $24-28$ (2006)

14) Bahrami Gh., Kiani A., J. Chromatogr. B, 835, 123-126 (2006).

15) Sagirli O., Cetin S. M., Onal A., J. Pharm. Biomed. Anal., 42, 618624 (2006).

16) "The United States Pharmacopeia," 30th revision, Asian Edition, United States Pharmacopeial Convention, Inc., Twinbrook Parkway, Rockville, MD, 2007, p. 2200.

17) Belal F., Abdine H., Al-Majed A., Khalil N. Y., J. Pharm. Biomed. Anal., 27, 253-260 (2002)

18) Abdellatef H. E., Khalil H. M., J. Pharm. Biomed. Anal., 31, 209214 (2003).

19) Sekar R., Azhaguvel S., J. Pharm. Biomed. Anal., 36, 663-667 (2004).

20) Zhou M., Wei G., Liu Y., Sun Y., Xiao Sh., Lu L., Liu Ch., Zhong D., J. Chromatogr. B, 798, 43-48 (2003).

21) Nicoli S., Santi P., J. Pharm. Biomed. Anal., 41, $994-997$ (2006).

22) Paraskevas G., Atta-Politou J., Koupparis M., J. Pharm. Biomed. Anal., 29, 865-872 (2002). 